\title{
AVALIAÇÃO DE DESEMPENHO DA CADEIA DE SUPRIMENTOS BALIZADA POR CRITÉRIOS DE COMPETITIVIDADE EMPRESARIAL
}

\section{A COMPETITIVE CRITERIA-BASED MODEL FOR ASSESSING SUPPLY CHAIN PERFORMANCE}

\author{
Diego Vinícius Souza de Souza*E-mail: dviniciuss@uol.com.br \\ Francisco José Kliemann Neto* E-mail: kliemann@producao.ufrgs.br \\ Michel José Anzanello* E-mail: anzanello@producao.ufrgs.br \\ *Universidade Federal do Rio Grande do Sul, Porto Alegre, RS
}

\begin{abstract}
Resumo: A competição de mercado faz com que as empresas busquem eficiência não somente dentro de seus limites físicos, mas em todos os elos da cadeia produtiva em que estão inseridas. A medição de desempenho da cadeia demanda indicadores integrados e que priorizem a avaliação conjunta do sistema em detrimento à análise de um processo específico. Este artigo propõe uma sistemática fundamentada em indicadores de competitividade empresarial para avaliar o desempenho de cadeias de suprimentos. Os diversos indicadores são agrupados em subgrupos associados a critérios de competitividade, e então hierarquizados através do método Analytic Hierarchy Process (AHP). Um índice geral de desempenho da cadeia é então gerado através da Função Preferência. A sistemática proposta viabiliza uma análise crítica de desempenho sob a ótica agregada e desagregada. A metodologia é ilustrada através de um estudo de caso em quatro empresas de logística do setor siderúrgico.
\end{abstract}

Palavras chave: Avaliação de desempenho empresarial. Gestão da cadeia de suprimentos. Indicadores de desempenho. Sistemas de produção

\begin{abstract}
In the highly competitive market, companies are required to focus on efficiency within and outside the organization. Performance measures must be able to evaluate the entire organization instead of a limited number of processes. This paper suggests a method to evaluate supply chain performance using competitive criteria. We first group several performance indices into clusters associated to several competitive criteria, and then order those indices using the Analytic Hierarchy Process (AHP). A consolidated index for measuring the company's performance is obtained by means of the Preference Function. The proposed method enables a critical analysis on both aggregated and disaggregated senses. We illustrate the proposed method on 4 logistics companies in a steel company.
\end{abstract}

Key words: Performance measurement. Supply Chain management. Performance indices. Manufacturing Systems.

\section{INTRODUÇÃO}

Uma organização empresarial não é mais vista de forma isolada, mas como parte de uma rede de empresas conectadas por uma sequência de atividades e decisões 
dentro e fora de suas fronteiras físicas (FU; PIPLAN, 2004). A gestão desta rede, ou cadeia, é norteada pelo aprimoramento dos processos produtivos com vistas a elevados padrões de qualidade e custos reduzidos (SIMCHI-LEVI, 2000). Esse processo de aprimoramento é usualmente balizado por critérios de competitividade empresarial.

Os critérios de competitividade formam um conjunto de prioridades que a empresa deve adotar no seu contexto de participante no mercado. Tais critérios incluem aspectos de custo, qualidade e flexibilidade, entre outros, e devem ser avaliados de forma integrada na busca de medidas que beneficiem a cadeia de forma equilibrada (SLACK; LEWIS, 2008). O uso de parâmetros de desempenho capazes de capturar tais critérios e traduzi-los em ações concretas configura-se como vital para a manutenção da cadeia de suprimentos.

A ineficiência ou falta de exatidão de métodos de avaliação sistêmica de desempenho é discutida desde a publicação do Balanced Scorecard (BSC) (KAPLAN; NORTON, 1997) até estudos mais recentes, nos quais uma das mais recorrentes discussões está relacionada à comunicação e ao controle da estratégia (MALINA; SELTO, 2002; DABHILAKAR; BENGTSSON, 2002). Muitos métodos são criticados por não serem capazes de desdobrar seus sistemas de indicadores para uma medição integrada de desempenho, além de apoiarem-se em orientações puramente operacionais ou estratégicas. As cadeias de suprimento, em especial, impõem um fator adicional de dificuldade: a diversidade na maturidade de gestão nas empresas constituintes da cadeia influencia diretamente no resultado do conjunto. O direcionador operacional passa a focar-se no nivelamento geral dos integrantes, o qual deve ser balizado por sistemáticas robustas e abrangentes de medição de desempenho. No entanto, percebe-se uma carência de sistemáticas capazes de avaliar a cadeia de forma abrangente e com base em uma ampla gama de critérios de competitividade empresarial.

Este artigo apresenta uma sistemática de avaliação de desempenho aplicada à gestão da cadeia de suprimentos com base em critérios de competitividade empresarial. Critérios de competitividade são inicialmente identificados, permitindo a geração de 
indicadores de desempenho associados a cada critério. Tais critérios e indicadores são avaliados hierarquicamente através do método Analytic Hierarchy Process (AHP), e então consolidados em um indicador geral de desempenho da cadeia de suprimento através da Função Preferência. Os resultados gerados permitem tanto uma avaliação panorâmica da cadeia (análise agregada), como uma avaliação focada em um critério de competitividade específico (análise desagregada).

O presente artigo inova ao estruturar uma sistemática de avaliação de desempenho desdobrada a partir dos critérios de competitividade empresarial, tendo como ponto de partida a premissa de eficiência e eficácia em relação a custo, qualidade, velocidade, confiabilidade e flexibilidade. $O$ artigo também adapta a Função Preferência, recurso tipicamente utilizado na área de controle de qualidade, para a consolidação de um indicador de desempenho da cadeia. Por fim, o método também contribui ao permitir a apresentação, de forma agregada e desagregada, dos indicadores aplicados ao contexto da gestão da cadeia de suprimentos.

O restante do artigo é organizado como segue. A seção 2 apresenta uma breve introdução aos modelos de medição de desempenho na cadeia de suprimentos, AHP e Função Preferência. Na seção 3 é apresentado o método para geração do Índice Geral de Desempenho para avaliação da cadeia de suprimentos. A seção 4 apresenta os resultados obtidos através da aplicação da metodologia proposta em uma indústria siderúrgica com vistas à avaliação do desempenho da logística de distribuição. A seção 5 traz a conclusão do artigo.

\section{REFERENCIAL TEÓRICO}

\subsection{Aspectos associados à gestão da cadeia de suprimentos}

Em uma típica cadeia de suprimentos, as matérias-primas são produzidas/extraídas e então compradas pelos segmentos de manufatura. $\mathrm{Na}$ sequência, os produtos são fabricados, transportados até os armazéns, e distribuídos aos comerciantes ou consumidores finais (SIMCHI-LEVI, 2000). Em geral, as operações 
de transporte, manuseio e armazenagem não agregam valor direto ao produto; tornálas mais eficientes, contudo, fará com que a sua participação no grau de valor percebido pelo cliente torne-se um fator determinante ou ganhador de pedido (BREWER; SPEH, 2000).

Uma das discussões fundamentais na cadeia de suprimentos reside no trade-off entre nível de serviço (geralmente representado pela disponibilidade de pontos de distribuição) e custos. Para Simchi-Levi (2000), as empresas devem balancear os custos operacionais e de capital inicial necessários para a abertura de uma nova operação, com as vantagens imediatas de estar mais próximo ao consumidor.

O nível de serviço também está intimamente ligado à política de estoque adotada pelos elementos da cadeia, sendo sua análise focada (I) no risco de ruptura na cadeia, e na (ii) possibilidade de ocorrência de efeito chicote. Uma ruptura é vista como o não atendimento de uma oportunidade de demanda, e, por conseqüência, de geração de receita (SIMCHI-LEVI, 2000). O efeito chicote, por sua vez, consiste na propagação da variação de demanda ao longo da cadeia, o qual implica no aumento dos tempos de entrega/produção, flutuações de preço e imprecisões no cálculo do lote econômico (CHEN; DREZNER; RYAN; SIMCHI-LEVI, 2000).

Outro aspecto fundamental na gestão da cadeia está na consolidação de alianças estratégicas. Mclvor (2001) destaca o desenvolvimento de relações de longo prazo em cadeias de suprimentos, tendo como referência a concepção de uma cadeia de suprimentos voltada para a redução de custos. O mesmo autor acrescenta que, como ponto de partida para o estabelecimento de alianças, as empresas devem revisar os seus sistemas de planejamento visando difundir os pontos positivos ao longo de sua estrutura física e de recursos humanos. Complementarmente, Simchi-Levi (2000) destaca que o objetivo maior para o estabelecimento de parcerias estratégicas é o de fortalecer a cadeia como um todo, atentando para não enfraquecer as competências centrais de uma determinada organização em detrimento das demais. 


\subsection{Avaliação de desempenho empresarial: Definição e Modelos}

A Avaliação de Desempenho Empresarial (ADE) tem como principal objetivo a mensuração do desempenho dos processos nas suas interfaces. Hronec (1993), Harrington (1965) e Harrington (1993) estabeleceram as bases conceituais para a discussão deste tema, evidenciando o impacto da ADE na sustentação do resultado das empresas.

As medidas de desempenho, entretanto, passaram por um cenário evolutivo nos últimos anos, passando de um viés voltado para medidas exclusivamente financeiras para uma visão de medição de desempenho nas dimensões competitivas de custo, prazo, qualidade, velocidade e confiabilidade (SLACK, 1993). Em um ambiente de cadeias de suprimentos, a implantação de indicadores de desempenho mostra-se como relevante, dado que múltiplos processos de empresas diferentes passam a interagir entre si (SIMCHI-LEVI, 2000).

O Balanced Scorecard (BSC) mostra-se como modelo genérico e amplamente utilizado no meio empresarial dado seu caráter com foco em processos (KAPLAN; NORTON, 1992). Para Kaplan e Norton (1997) o BSC tem como objetivos (i) fortalecer a visão estratégica, comunicando objetivos e medidas estratégicas de forma visual, (ii) estabelecer metas alinhadas as iniciativas corporativas, e (iii) melhorar o feedback ao longo de toda a organização. Adicionalmente, o BSC permite a análise do processo através de quatro perspectivas centrais - financeira, cliente, processos internos e crescimento e aprendizado (KAPLAN; NORTON, 1997; EILAT et al., 2008). As medidas de desempenho do BSC são coordenadas pela utilização de indicadores traçados a partir dos objetivos estratégicos organizacionais e embasados em um sistema de metas de desempenho.

Por sua vez, o modelo SCOR (Supply Chain Operations Reference Model) faz uso de comparativos entre empresas e de interavaliações para a melhoria de desempenho de cadeias de suprimentos. O SCOR é um modelo de caráter operacional e com foco no aprendizado das companhias em relação aos processos internos e externos ao seu ramo de atuação (STEWART, 1997). O SCOR utiliza-se de conceitos 
de reengenharia, benchmarking e medição de desempenho (SUPPLY-CHAIN COUNCIL, 2005) para a sua efetivação nas organizações. Para Stewart (1997), o SCOR proporciona às empresas a avaliação de processos, a comparação de desempenho com outras empresas através do benchmarking e a utilização de ferramentas de tecnologia da informação para a gestão dos processos. Maiores detalhes sobre SCOR podem ser obtidas em Huan et al. (2004), Lockamy e McCormack (2004) e Blecken et al. (2009).

Por fim, o Global Supply Chain Forum Model (GSCF) é um modelo com enfoque mais estratégico, estruturado para integrar processos desde o usuário final até os fornecedores em uma cadeia de suprimentos. O GSCF é desdobrado em oito áreas (ver LAMBERT, 2004), as quais são operacionalizadas com foco especial no desempenho com vistas ao retorno para clientes e investidores. Maiores detalhes sobre o GSCF são apresentados em Lambert (2004) e Chow et al. (2008).

Os modelos descritos até então apresentam caráter complementar, dado que sua escolha se dá pelo seu maior grau de aderência ao nível de maturidade de gestão ou contexto de mercado no qual a empresa está inserida. Contudo, tais modelos não oferecem uma avaliação de desempenho suficientemente abrangente em alguns processos empresariais (caso da gestão da cadeia de suprimentos), visto que tradicionalmente incorporam limitado número de indicadores e/ou não contemplam a influência de critérios de competitividade em suas estruturas. Adicionalmente, os modelos não permitem uma visão geral do desempenho do processo, dado que apresentam somente os indicadores com seus resultados individuais.

\subsection{AHP - Analytical Hierarchical Process}

O AHP é um método de avaliação multicriterial amplamente utilizado na tomada de decisão empresarial, o qual foi descrito por Saaty (2008). Essa abordagem tem como foco criar a hierarquia entre atributos e parâmetros, estabelecendo entre eles graus de importância ou de relevância. Saaty (2008), na descrição do método AHP, 
estabelece que o estabelecimento da hierarquização dos parâmetros deve ser realizada sob a lógica de comparações pareadas, as quais são exemplificadas na tabela 1:

Tabela 1 - Processo de comparação pareada

\begin{tabular}{ccccc}
\hline Atributos & $\boldsymbol{A}_{1}$ & $\boldsymbol{A}_{\mathbf{2}}$ & $\boldsymbol{A}_{\mathbf{3}}$ & $\boldsymbol{A}_{\boldsymbol{n}}$ \\
\hline $\boldsymbol{A}_{1}$ & 1 & $a_{12}$ & $a_{13}$ & $a_{1 n}$ \\
$\boldsymbol{A}_{2}$ & $1 / \mathrm{a}_{12}$ & 1 & $a_{23}$ & $a_{2 n}$ \\
$\boldsymbol{A}_{3}$ & $1 / \mathrm{a}_{13}$ & $1 / \mathrm{a}_{23}$ & 1 & $a_{3 n}$ \\
$\boldsymbol{A}_{\boldsymbol{n}}$ & $1 / \mathrm{a}_{1 \mathrm{n}}$ & $1 / \mathrm{a}_{2 \mathrm{n}}$ & $1 / \mathrm{a}_{3 \mathrm{n}}$ & 1 \\
\hline
\end{tabular}

Após a realização das comparações pareadas, faz-se necessária a normalização das colunas para verificar a contribuição de cada atributo ao modelo de comparação, abordada detalhadamente por Saaty (2008). Adicionalmente, é verificado o índice de consistência, que avalia a coerência entre as avaliações realizadas, o qual foi também foi desdobrado por Saaty (2008) e tem seus resultados recomendados no intervalo entre 0 e 0,10 .

De forma geral, o AHP é utilizado nos processos de tomada de decisão empresarial, norteando os processos empresariais através da análise simultânea de múltiplos parâmetros e/ou atributos (CHENG, 2006). Finalmente, é válido lembrar que o AHP possui aplicação prática bastante simplificada, sendo um dos métodos de avaliação multicriterial mais utilizado no meio empresarial.

\subsection{Função Preferência}

A Função Preferência (FP) tem como objetivo colocar variáveis diferentes de modo consolidado. Essas variáveis podem ser apresentadas sob a forma de indicadores ou métricas de processos empresariais, tanto no âmbito da gestão quanto no âmbito das operações. Alguns estudos têm sido conduzidos com o objetivo de avaliar diferentes aplicações da FP, a qual está relacionada também aos problemas de 
análise multicriterial e de otimização de processos tais como Trautmann e Mehnen (2008), Jeong e Kim (2009) e Lee e Kim (2007).

A FP tem como principal objetivo colocar os valores de variáveis de resposta em uma escala de 0 a 1 , seguindo a lógica de transformar uma média estimada de resposta $\left(\hat{\omega}_{\mu i}\right)$ em um fator individual de desempenho desejado $\left(d_{\mu i}\right)(i=1,2,3, \ldots, \mathrm{n})$. Agregando-se os fatores individuais de desempenho em um único índice $(D)$, obtêm-se novamente um valor que varia em uma escala de 0 a 1 (LEE, KIM; 2007). Desta forma, passa a ser o objetivo central desta abordagem encontrar valores para as variáveis de entrada $\boldsymbol{X}^{*}$ que otimizem o índice consolidado (agregado) de desempenho $(D)$. Lee e Kim (2007) apontam a equação 1 como a mais tradicional forma de expressão da FP:

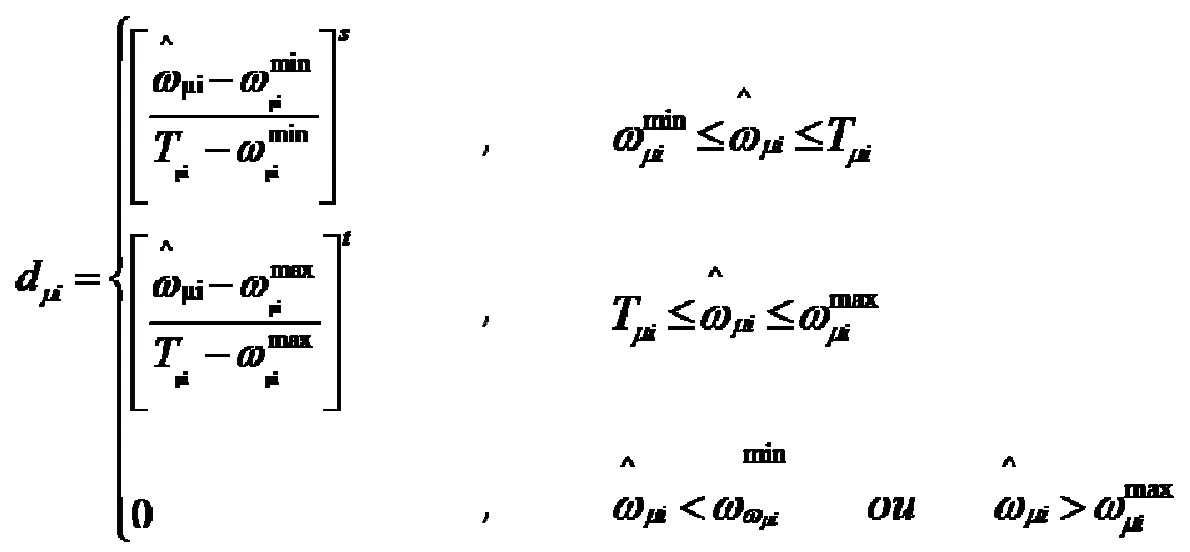

Na equação, $T \mu i, \omega_{\mu i}^{\max }$ e $\omega_{\mu i}^{\text {min }}$ definem o valor alvo, o valor máximo e o valor mínimo, respectivamente, para os limites de $\widehat{\omega}_{\mu i}$. Adicionalmente, os valores de $s$ e $t$ são constituídos por parâmetros de forma da FP (LEE, KIM; 2007). Para a obtenção do valor agregado $D$, faz-se uma média geométrica dos fatores conforme a equação 2 :

$$
\mathrm{D}=\left(d_{\mu 1} \times d_{\mu 2} \times d_{\mu 3} \times \ldots \times d_{\mu n}\right)^{1 / n}
$$




\section{MÉTODO}

A sistemática proposta é operacionalizada em cinco etapas. A etapa 1 aborda aspectos conceituais e foca na formação da equipe e mapeamento do processo. As etapas 2 a 5 têm caráter operacional e são sistematizadas por intermédio da manipulação dos indicadores e índices gerados através da avaliação de especialistas de processo. Essas etapas incluem a definição do conjunto de parâmetros de desempenho, construção da árvore de relações para o método AHP, cálculo de um índice geral de desempenho através da função preferência e consolidação da análise crítica dos outputs fornecidos. Essas etapas são descritas na próxima seção.

\section{Etapa 1 - Estruturação da equipe de especialistas de processo}

Esta etapa inicia com a estruturação de uma equipe que forneça informações quantitativas e qualitativas acerca da cadeia de suprimentos em análise. Tais informações incluem dimensão da cadeia, intensidade de interação entre os níveis, valores financeiros envolvidos e peculiaridades dos processos desenvolvidos.

A equipe deve ser composta por um grupo multifuncional de profissionais com expertise no processo de gestão da cadeia e de avaliação de desempenho empresarial. Além disso, recomenda-se que os membros possuam visão global do negócio com vistas à validação da sistemática. Os perfis recomendados incluem pessoal com expertise em gestão de cadeias e avaliação de desempenho, membros da alta gerência, equipe operacional e fornecedores/clientes.

\section{Etapa 2 - Definição e hierarquização dos critérios de competitividade}

Esta etapa inicia com a identificação de Critérios de Competitividade (CC) aplicáveis ao processo de avaliação de desempenho da cadeia. Os CCs são levantados pelos especialistas de processo avaliando-se aspectos como $(I)$ grau de maturidade em gestão empresarial das empresas que fazem parte do escopo avaliado (fornecedores e clientes); (ii) pontos críticos, tais como índice de dependência econômica e/ou de estoque de peças ao longo da cadeia; e (iii) desempenho prévio das empresas pertencentes à cadeia quanto ao atendimento de parâmetros ligados aos critérios de 
competitividade empresarial. Os CCs podem ser elencados através de técnicas de grupo, como Delphi e entrevistas estruturadas. Maiores detalhes acerca da fundamentação dos CCs podem ser encontrados em Slack e Lewis (2008).

$\mathrm{Na}$ sequência, cada CC é mensurado através de indicadores de desempenho apropriados, os quais são detalhados na etapa 3 deste método. Este estudo sugere cinco CCs de larga utilização em cadeias de suprimentos, bem como características que viabilizem a medição desses CCs via indicadores (SLACK e LEWIS, 2008): (I) Qualidade - dimensões da preservação do desempenho funcional, conformidade e especificações, estética, embalagem e qualidade percebida; (ii) Velocidade - tempos de aquisição, produção e entrega; (iii) Confiabilidade - percepção e confiança do cliente quanto ao grau de acerto promovido pela empresa de forma geral (aquisição, produção e entrega); (iv) Flexibilidade - grau de resposta que a empresa pode operar dadas variações no escopo, demanda e/ou especificações promovidas pelo mercado ou pela cadeia; e $(v)$ Custo - relação entre a margem de contribuição, parcela fixa em relação à parcela variável no sistema de custeio unitário do produto e à utilização da estrutura. Outros CCs podem ser incluídos na análise, de acordo com peculiaridades da cadeia em questão.

Por fim, hierarquizam-se os CCs elencados acima através do método AHP. Os CCs são arranjados de acordo com a matriz apresentada na tabela 1 (os CCs substituem os atributos neste quadro). A priorização dos CCs é expressa através do peso relativo médio do critério $i\left(P C C_{i}\right)$; os $P C C_{i}$ para os I critérios são consolidados em um vetor PCC (segunda coluna do Quadro 1). O nível de consistência do processo é verificado através do cálculo do índice de consistência gerado pelo método AHP (ver SAATY, 2008). Caso tal índice esteja acima de 0,10 , as avaliações realizadas devem ser refeitas, até atingir resultados entre 0 e 0,10.

\section{Etapa 3 - Definição e hierarquização dos indicadores de desempenho}

Nesta etapa, geram-se indicadores de desempenho para descrição quantitativa de cada CC gerado na Etapa 2. O Quadro 1 lista os itens considerados mínimos para a consolidação de um indicador, apresentando um exemplo de indicador para o CC 
Qualidade. Vale salientar que é possível suprimir ou substituir indicadores de acordo com as particularidades do processo ou cenário no qual a empresa estiver inserida, desde que os requisitos para utilização de indicadores de desempenho sejam atendidos.

Quadro 1 - Itens mínimos necessários para a definição de um indicador

\begin{tabular}{|l|}
\hline Nome do indicador: Reclamações de clientes \\
\hline Critério de Competitividade (CC) ligado ao indicador: qualidade \\
\hline $\begin{array}{l}\text { Descrição formal (para que serve): Registra as reclamações de clientes } \\
\text { entrega, etc) }\end{array}$ \\
\hline Periodicidade de medição: mensal \\
\hline Fórmula de cálculo: $\sum \boldsymbol{R C}$ (onde $\boldsymbol{R C}=$ Reclamações de Clientes) \\
\hline Unidade de medida: no de reclamações/mês \\
\hline Valor-alvo ou meta: 63 reclamações/mês \\
\hline Tipo (nominal é melhor, maior é melhor, menor é melhor): menor é melhor \\
\hline Limite de especificação superior (LES) - 69,3 reclamações/mês \\
\hline Limite de especificação inferior (LEl) - não aplicável \\
\hline
\end{tabular}

Fonte: Autores

$\mathrm{Na}$ sequência, estabelecem-se relações hierarquizadas entre os indicadores de desempenho gerados para cada CC através do método AHP. Os indicadores de cada CC são identificados através da notação $\mathrm{PI} X_{j}$, onde $X_{j}$ denota o $j$-ésimo indicador do critério $X$ (por exemplo, $\mathrm{PI}_{3}$ refere-se ao terceiro indicador do $\mathrm{CC} \underline{\mathrm{Q} u a l i d a d e) . ~ T a i s ~}$ informações são apresentadas na terceira coluna do Quadro 3. Repare que cada CC pode apresentar um tamanho distinto para PIX por conta do diferente número de indicadores atrelados aquele critério.

\section{Etapa 4 - Consolidação dos vetores finais de desempenho}

Nesta etapa ocorre a integração entre os vetores PCC e PIX. A idéia é calibrar a priorização de cada indicador pela priorização do critério de competitividade associado. Para tanto, cada elemento do vetor PCC, representando a priorização de um CC, é multiplicado pelos valores de priorização dos indicadores daquele CC em PIX. O vetor resultante é denominado Vetor de Desempenho (VD), conforme apresentado na última coluna do Quadro 2. 
Quadro 2 - Consolidação do Vetor de Desempenho (VD)

\begin{tabular}{|c|c|c|c|}
\hline $\begin{array}{c}\text { Critérios de } \\
\text { Competitividade } \\
\text { (CC) }\end{array}$ & $\begin{array}{l}\text { Priorização do } \\
\text { CC } \\
\text { (vetor PCC) }\end{array}$ & $\begin{array}{c}\text { Priorização dos } \\
\text { Indicadores de } \\
\text { desempenho em cada CC } \\
\text { (vetor PIX) }\end{array}$ & $\begin{array}{l}\text { Vetor de Desempenho } \\
\text { (VD) }\end{array}$ \\
\hline Qualidade & $\mathrm{PCC}_{1}$ & $\begin{array}{c}\mathrm{PIQ}_{1} \\
: \\
\mathrm{PIQ} q \\
\end{array}$ & $\begin{array}{c}\mathrm{PCC}_{1} \times \mathrm{PIQ}_{1} \\
: \\
\mathrm{PCC}_{1} \times \mathrm{PIQq}\end{array}$ \\
\hline Velocidade & $\mathrm{PCC}_{2}$ & $\begin{array}{c}\operatorname{PIV}_{1} \\
\vdots \\
\text { PIVv }\end{array}$ & $\begin{array}{c}\mathrm{PCC}_{2} \times \mathrm{PIV}_{1} \\
: \\
\mathrm{PCC}_{2} \times \mathrm{PIV} v^{2}\end{array}$ \\
\hline Confiabilidade & $\mathrm{PCC}_{3}$ & $\begin{array}{l}\mathrm{PIC}_{1} \\
\vdots \\
\mathrm{PICC}\end{array}$ & $\begin{array}{c}\mathrm{PCC}_{3} \times \mathrm{PIC}_{1} \\
: \\
\mathrm{PCC}_{3} \times \mathrm{PICC}^{2}\end{array}$ \\
\hline Flexibilidade & $\mathrm{PCC}_{4}$ & $\begin{array}{l}\mathrm{PIF}_{1} \\
\vdots \\
\mathrm{PIFf}\end{array}$ & $\begin{array}{c}\mathrm{PCC}_{4} \times \mathrm{PIF}_{1} \\
: \\
\mathrm{PCC}_{4} \times \mathrm{PIFf}\end{array}$ \\
\hline Custo & $\mathrm{PCC}_{5}$ & $\begin{array}{l}\mathrm{PIT}_{1} \\
: \\
\mathrm{PIT} t\end{array}$ & $\begin{array}{c}\mathrm{PCC}_{5} \times \mathrm{PIT}_{1} \\
: \\
\mathrm{PCC}_{5} \times \mathrm{PIT} t\end{array}$ \\
\hline
\end{tabular}

Fonte: Autores

\section{Etapa 5 - Geração do Índice Global de Performance (IGP) e Análise crítica da sistemática}

Esta etapa inicia com a avaliação do desempenho dos indicadores utilizando a Função Preferência (FP), conforme a equação (5). O FP posiciona o indicador quanto ao seu potencial de melhoria ou de manutenção do resultado obtido, sendo representado no intervalo [0, 1]. O Índice Geral (IG) para cada indicador é gerado através da multiplicação de VD pelo respectivo FP, gerando-se um total de $q+v+c+f+t$ IG's. O IGP, contido no intervalo [0,1], é consolidado através da soma dos IG's dentro de cada CC, visando quantificar o desempenho daquele CC. Nesse contexto, embora o índice IG esteja consolidado, a base de dados poderá ser acessada a qualquer momento para análise de um indicador de desempenho específico (análise desagregada).

O valor de IGP é então utilizado para avaliar o processo. A Tabela 2 apresenta uma escala sugerida com base na experiência do autor e nos estudos de Trautman e Weihs (2005) e Derringer e Suich (1980). 
Tabela 2 - Valores de referência para o IGP

\begin{tabular}{cc}
\hline Faixa de Variação do IGP & Desempenho do processo \\
\hline 0,81 a 1,00 & Acima do esperado \\
0,61 a 0,80 & Satisfatório \\
0,41 a 0,60 & Médio \\
0,21 a 0,40 & Insatisfatório \\
0,00 a 0,20 & Abaixo do esperado \\
\hline
\end{tabular}

Fonte: Autores

Tendo-se em vista que a grande maioria dos indicadores pode ser aferida mensalmente, o método proposto possibilita ainda a geração dos IGs para diversos períodos de análise. Essa informação pode ser consolidada em um gráfico temporal. Alternativamente, os valores de IGP podem ser arranjados em um gráfico de radar, o qual permite uma comparação visual do desempenho em cada CC para um período específico.

A proposição de melhorias em torno de um CC de baixo desempenho dever ser priorizada, visto que sua ineficiência aumenta as chances de uma empresa tornar-se pouco competitiva e perder significativas fatias de mercado. Ações de melhoria podem ser definidas utilizando-se questionamentos no formato $5 \mathrm{~W} 2 \mathrm{H}$ (detalhes sobre a metodologia 5W2H são suprimidos por limitações de espaço).

\subsection{Estudo de caso}

O método proposto é aplicado em quatro empresas do setor logístico de uma empresa siderúrgica. Tal setor compreende atividades de manuseio, armazenagem, carregamento e gestão do transportes de produtos. Esse setor interage com diversas áreas de negócio da empresa, em especial com vendas, programação e controle, produção, clientes e com o mercado transportador. Em termos gerais, a distribuição tem como direcionadores a manutenção e melhoria do nível de serviço ao menor custo possível.

A estruturação da equipe de especialistas incluiu os seguintes profissionais: (i) 2 Supervisores de Logística - profissionais com experiência de mais de 7 anos no processo e expertise em gestão de cadeia de suprimentos, com excelente visão operacional e tática da estratégia para distribuição; (ii) 3 Analistas de Logística - 
profissionais com mais de 3 anos de experiência na gestão do processo e expertise em avaliação de desempenho; (iii) 1 Gerente de Logística - profissional com mais de 30 anos de experiência na área, incluindo inúmeras visitas ao exterior para conhecer melhores práticas; (iv) 2 Gestores de Transportadora - profissionais representado fornecedores e/ou clientes, com mais de 10 anos de experiência no mercado transportador; e (v) 5 Operadores de Logística - profissionais com mais de 10 anos em armazenagem e carregamento de materiais siderúrgicos.

$\mathrm{Na}$ sequência, são selecionados 28 indicadores de desempenho sugeridos pela literatura para quantificar os 5 critérios de competitividade (CC) analisados, conforme apresentado na Figura 1. A descrição formal de cada indicador, bem como suas especificações, é apresentada no Anexo 1.

Figura 1 - Indicadores de desempenho para cada CC

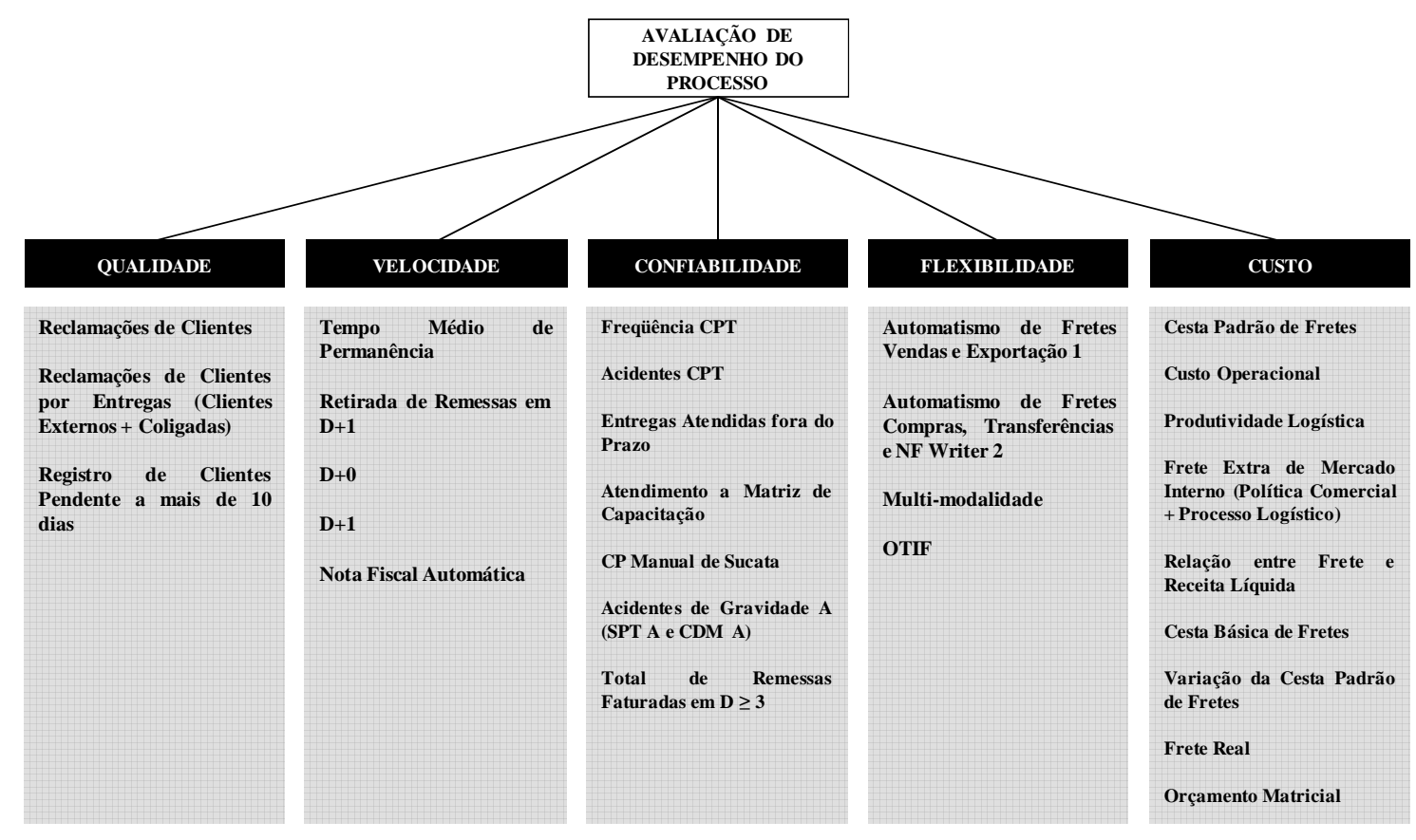

Fonte: Autores

A relação hierárquica entre os CCs gerada pela matriz AHP, bem como o vetor de priorização PCC, é apresentada na Tabela 3. Priorização é dada aos CCs Confiabilidade e Custo. A razão de consistência obtida é de 0,06. 
Tabela 3 - Critérios de Competitividade (CC) e vetor PCC

\begin{tabular}{|l|c|c|c|c|c|c|}
\hline & Qualidade & Velocidade & Confiabilidade & Flexibilidade & Custo & PCC \\
\hline Qualidade & 1,00 & 0,50 & 0,50 & 1,00 & 0,25 & 0,1047 \\
\hline Velocidade & 2,00 & 1,00 & 0,50 & 3,00 & 1,00 & 0,2251 \\
\hline Confiabilidade & 2,00 & 2,00 & 1,00 & 3,00 & 1,00 & 0,2965 \\
\hline Flexibilidade & 1,00 & 0,33 & 0,33 & 1,00 & 1,00 & 0,1231 \\
\hline Custo & 4,00 & 1,00 & 1,00 & 1,00 & 1,00 & 0,2507 \\
\hline
\end{tabular}

Fonte: Autores

Os indicadores associados a cada CC são então hierarquizados através do método AHP, gerando-se o vetor de priorização dos indicadores, PIX. Os valores de PIQ (para o CC Q Qualidade) são apresentados na Tabela 4, sendo que o indicador "Registro de clientes pendentes a mais de 10 dias" deve ser priorizado. A priorização dos indicadores dos demais CCs é obtida de forma análoga.

Tabela 4 - Indicadores do CC Qualidade e Vetor PIQ

\begin{tabular}{|l|c|c|c|c|}
\hline & $\begin{array}{c}\text { Reclamações de } \\
\text { Clientes }\end{array}$ & $\begin{array}{c}\text { Reclamações de Clientes por Entregas } \\
\text { (Clientes Externos + Coligadas) }\end{array}$ & $\begin{array}{c}\text { Registro de Clientes } \\
\text { Pendente a mais de 10 dias }\end{array}$ & PIQ \\
\hline Reclamações de Clientes & 1,00 & 0,50 & 0,33 & 0,1593 \\
\hline $\begin{array}{l}\text { Reclamações de Clientes por Entregas } \\
\text { (Clientes Externos + Coligadas) }\end{array}$ & 2,00 & 1,00 & 0,33 & 0,2519 \\
\hline $\begin{array}{l}\text { Registro de Clientes Pendente a mais } \\
\text { de 10 dias }\end{array}$ & 3,00 & 3,00 & 1,00 & 0,5889 \\
\hline
\end{tabular}

Fonte: Autores

O Vetor de Desempenho (VD) é consolidado a partir dos vetores PCC e PIX, conforme apresentado na Tabela 5. A terceira coluna apresenta um código de identificação dos indicadores de desempenho. 
Tabela 5 - Vetores PCC, PIX e VD

\begin{tabular}{|c|c|c|c|c|}
\hline $\begin{array}{c}\text { Critérios de } \\
\text { Competitividade }\end{array}$ & PCC & $\begin{array}{c}\text { Indicadores de } \\
\text { desempenho (código) }\end{array}$ & PIX & $\begin{array}{c}\text { Vetor de } \\
\text { Desempenho (VD) }\end{array}$ \\
\hline \multirow{3}{*}{ Qualidade } & \multirow{3}{*}{0,1047} & 3 & 0,1593 & 0,0167 \\
\hline & & 11 & 0,2519 & 0,0264 \\
\hline & & 19 & 0,5889 & 0,0616 \\
\hline \multirow{5}{*}{ Velocidade } & \multirow{5}{*}{0,2251} & 7 & 0,1356 & 0,0305 \\
\hline & & 8 & 0,1724 & 0,0388 \\
\hline & & 10 & 0,3585 & 0,0807 \\
\hline & & 15 & 0,1001 & 0,0225 \\
\hline & & 23 & 0,2335 & 0,0526 \\
\hline \multirow{7}{*}{ Confiabilidade } & \multirow{7}{*}{0,2965} & 1 & 0,1566 & 0,0464 \\
\hline & & 2 & 0,1854 & 0,0550 \\
\hline & & 9 & 0,1426 & 0,0423 \\
\hline & & 12 & 0,1559 & 0,0462 \\
\hline & & 16 & 0,0501 & 0,0148 \\
\hline & & 18 & 0,0594 & 0,0176 \\
\hline & & 26 & 0,2500 & 0,0741 \\
\hline \multirow{4}{*}{ Flexibilidade } & \multirow{4}{*}{0,1231} & 13 & 0,1345 & 0,0166 \\
\hline & & 14 & 0,2422 & 0,0298 \\
\hline & & 20 & 0,2978 & 0,0366 \\
\hline & & 21 & 0,3255 & 0,0401 \\
\hline \multirow{9}{*}{ Custo } & \multirow{9}{*}{0,2507} & 4 & 0,0862 & 0,0216 \\
\hline & & 5 & 0,0926 & 0,0232 \\
\hline & & 6 & 0,1240 & 0,0311 \\
\hline & & 17 & 0,1349 & 0,0338 \\
\hline & & 22 & 0,0697 & 0,0175 \\
\hline & & 24 & 0,0849 & 0,0213 \\
\hline & & 25 & 0,1229 & 0,0308 \\
\hline & & 27 & 0,1852 & 0,0464 \\
\hline & & 28 & 0,0996 & 0,0250 \\
\hline
\end{tabular}

Fonte: Autores

$\mathrm{Na}$ sequência, os indicadores são confrontados com seus limites através da Função Preferência. Os resultados são descritos nas colunas FP do Anexo 2. Com vistas a uma análise mais dinâmica, o desempenho do processo foi avaliado para o período de janeiro a julho de 2009. Por fim, consolidam-se os valores mensais de IGP, gerados a partir do cruzamento de VD com os valores obtidos pela FP durante os meses de análise e apresentada no Anexo 2. 
A etapa final inicia com uma análise agregada do IGP, passando em seguida para níveis de maior desagregação. A Figura 2 ilustra o IGP do processo nos meses avaliados, sendo março o mês de melhor performance.

Figura 2 - IGP para os meses de janeiro/09 a junho/09

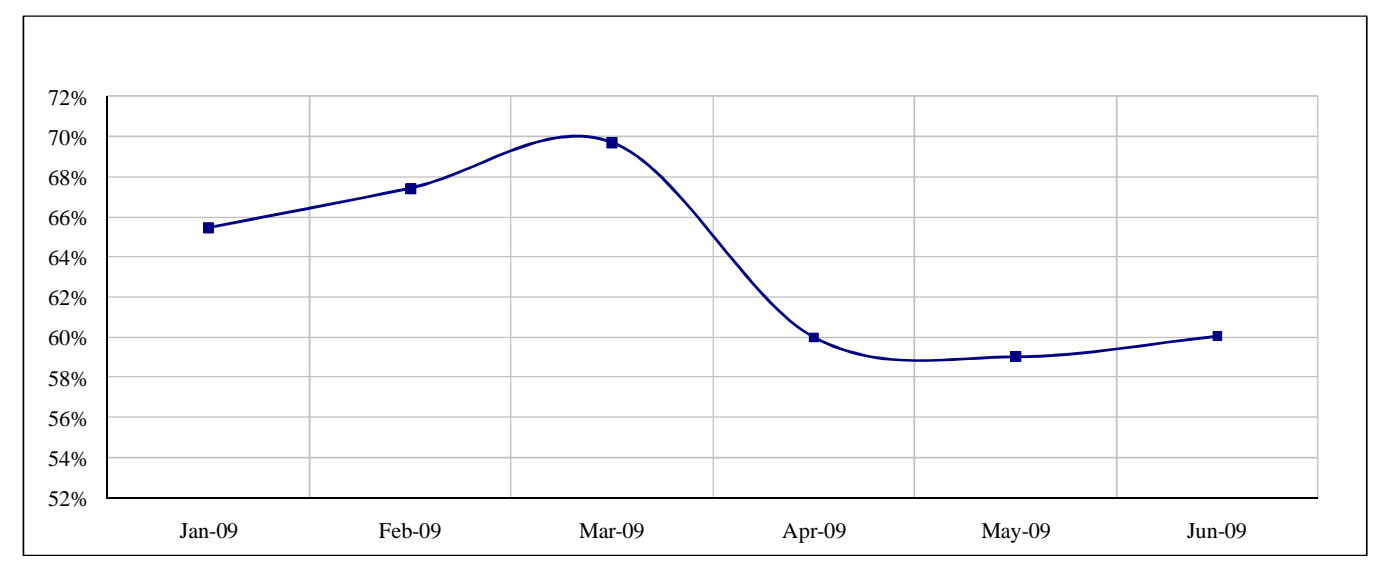

Fonte: Autores

Gráficos de radar são gerados utilizando-se os IGPs dos meses de performance mais forte (março/09) e mais fraca (maio/09), conforme apresentado nas Figuras 3 e 4.

Figura 3 - IGP março 09
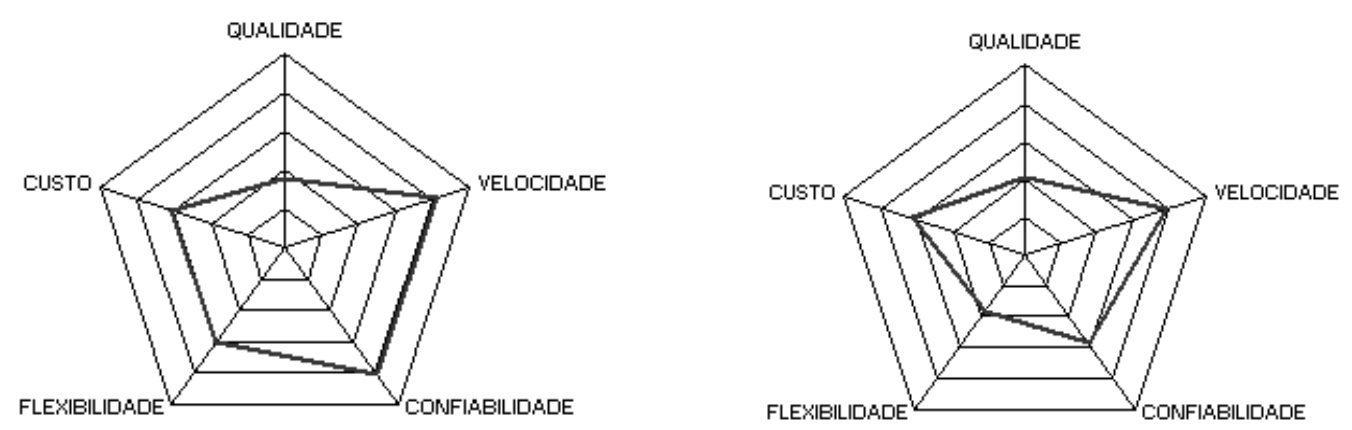

Fonte: Autores

Figura 4: IGP maio 09

Tais resultados são então analisados pela equipe de especialistas e razões para os diversos desempenhos são listadas. O mês de maio, por exemplo, apresentou fraca resposta em relação aos CCs Confiabilidade e Flexibilidade. Em termos de confiabilidade, percebeu-se que a resposta do indicador "Total de Remessas Faturadas 
em $D \geq 3$ " teve melhor desempenho e foi fundamental para direcionar o melhor desempenho de março. Em relação ao CC Flexibilidade, verificou-se que a medida do indicador "Multi-modalidade" foi superior em desempenho e contribuiu, de forma sensível, para o melhor desempenho de março.

Ao final, é construído um plano de ação, norteado por questionamentos no formato $5 \mathrm{~W} 2 \mathrm{H}$, com o objetivo de reduzir e/ou eliminar possíveis fraquezas (percebidas como riscos) advindas do processo analítico. O plano de ação é suprimido por restrições de espaço.

\section{CONCLUSÃO}

Os elevados níveis de competição, aliados à constante necessidade de resultados eficientes, demandam ferramentas precisas para a análise de desempenho empresarial. O intenso movimento de integração das empresas faz com que medidas de desempenho aplicadas de forma isolada em uma empresa mostrem-se ineficientes, visto que o desempenho da cadeia deve ser medido de forma global e não local. $A$ incorporação de preceitos de competitividade empresarial torna-se fundamental, visto que norteiam a tomada de decisão e o planejamento estratégico.

Este artigo apresenta uma sistemática de avaliação de desempenho aplicada à gestão da cadeia de suprimentos com base nos critérios de competitividade empresarial. Critérios de competitividade e indicadores de desempenho associados à gestão da cadeia de suprimentos são identificados, e então avaliados hierarquicamente através do método AHP. Na sequência, gera-se um indicador geral de desempenho da cadeia de suprimento através da Função Preferência. Os resultados gerados permitem avaliar a cadeia de forma agregada (todos os critérios consolidados), ou de forma desagregada (análise focada em um indicador de desempenho específico). O método, quando aplicado em um estudo de caso da indústria siderúrgica, gerou resultados coerentes com a avaliação da alta gerência.

Desdobramentos futuros acerca do tema incluem a utilização de ferramentas de seleção de variáveis para a identificação dos indicadores mais relevantes a serem utilizados na metodologia. Assume-se que a presença de indicadores de baixa 
relevância, ou oriundos de informações de baixa confiança, possa distorcer a importância dos critérios de competitividade e conduzir a conclusões imprecisas.

\section{REFERÊNCIAS}

BLECKEN, A.; HELLINGRATH, B.; DANGELMAIER, W.; SCHULZ, S. A humanitarian supply chain process reference model. International Journal of Services Technology and Management, v.12, n.4, 391-413, 2009.

BREWER, P. C.; SPEH, T. W. Using the balanced scorecard to measure supply-chain performance. Journal of Business Logistics, v. 21, n. 1, p. 75-93, 2000.

CHEN, F.; DREZNER, Z.; RYAN, J. K.; SIMCHI-LEVI, D. Quantifying the bullwhip effect in a simple supply chain: the impact of forecasting, lead times, and information.

Management Science, v. 46, n. 3. p. 436-443, 2000.

CHENG, H.; ZHANG, D.; YE, M.; XIA, J. Competitive capability evaluation for middle and smal enterprises in regional industries. The Business Review, Cambridge, v. 5, n. 2, p. 104, 2006.

CHOW, W.; MADU, C.; KUEI, C.; LU, M.; LIN, C.; TSENG, H. Supply chain management in the US and Taiwan: An empirical study. Omega, v. 36, n. 5, p.665-679, 2008.

DABHILAKAR, M. AND BENGTSSON, L. The Role of Balanced Scorecard in Manufacturing: a Tool for Strategically Aligned Work on Continuous Improvements in Production Teams. Performance Measurement and Management Control: a Compendium of Research.., Oxford, UK: Elsevier Science Ltd, p. 181-208, 2002.

DERRINGER, G.C.; SUICH, D. Simultaneous optimization of several response variables, Journal of Quality Technology, v.12, n. 4, p. $214-219,1980$.

EILAT, H.; GOLANY, B.; SHTUB, A. R\&D project evaluation: an integrated DEA and balanced scorecard approach. Omega, v.36, n.5, p.895-912, 2008.

FU, Y.; PIPLANI, R. Supply-side collaboration and its value in supply chains. European Journal of Operational Research, v. 152, p. 281-288, 2004.

HARRINGTON, E.C. The desirability function. Industrial Quality Control. v. 21, p. 494498, Apr. 1965. 
HARRINGTON, H. J. Aperfeiçoando processos empresariais. São Paulo: Makron Books, 1993.

HRONEC, S. M.; ARTHUR ANDERSEN CO. Vital signs: using quality, time and cost performance measurement to chart your company's future. New York: American Management Association, 1993.

HUAN, S. A review and analysis of supply chain operations reference (SCOR) model. Supply Chain Management, v. 9, n. 1, p. 23-29, 2004.

JEONG, I.; KIM, K. An interactive desirability function method to multiresponse optimization. European Journal of Operational Research, v. 195, n. 2, p. 412-426, 2009.

KAPLAN, R. S.; NORTON, D. P. A estratégia em ação: balanced scorecard. Tradução: Luiz E. T. Frazão Filho. Rio de Janeiro: Campus. Tradução de: The balanced scorecard, 1997.

KAPLAN, R. S.; NORTON, D. P. The balanced scorecard: measures that drive performance. Harvard Business Review, v. 70, n. 1, p. 71-79, 1992.

LAMBERT, D. M. Supply Chain Management. Supply Chain Management Review; Sep 2004, p. 18-26.

LEE, M.; KIM, K. Expected desirability function: consideration of both location and dispersion effects in desirability function approach. Quality Technology \& Quantitative Management, v. 4, n. 3, p. 365-377, 2007.

LOCKAMY III, A.; McCORMACK, K. Linking SCOR planning practices to supply-chain performance. International Journal of Operations \& Production Management, v. 24, n. 12, p. 1192-1218, 2004.

MALINA, M.A.; SELTO, F.H. Communicating and controlling strategy: an empirical study of the effectiveness of the balanced scorecard. Journal of Management Accounting Research, v. 13, 2002.

McIVOR, R. Lean supply: the design and cost reduction dimensions. European Journal of Purchasing \& Supply Management, v.7, p. 227-242, 2001

SAATY, T. L. Relative measurement and its generalization in decision making: why pairwise comparisons are central in mathematics for the measurement of intangible factors - the analytic hierarchy/network process. Revista Real Academia de Ciencias Exactas, Físicas y Naturales, v. 102, n. 2, p. 251-318, 2008.

SIMCHI-LEVI, D. Designing and Managing the Supply-Chain. United States of America: McGraw-Hill, 2000. 
SLACK, N. Vantagem competitiva em manufatura: atingindo competitividade em manufatura. São Paulo: Ed. Atlas, 1993, 193 p.

SLACK, N.; LEWIS, M. Operations Strategy. 2nd Edition, England, 2008.

STEWART, G. Supply-Chain Operations Reference Model (SCOR): The First CrossIndustry Framework for Integrated Supply-Chain Management. Logistics Information Management, 1997.

SUPPLY-CHAIN COUNCIL. Supply-Chain Operations Reference-model - SCOR version 7.0, 2005.

TRAUTMANN, H.; MEHNEN, J. Preference-Based Pareto-Optimization in Certain and Noisy Environments. Engineering Optimization, v. 41, p. 23-28, 2008.

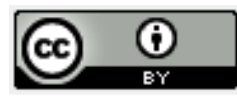

Artigo recebido em 12/04/2011 e aceito para publicação em 17/08/2011. 
ANEXO 1- INDICADORES DE DESEMPENHO UTILIZADOS NA ANÁLISE

\begin{tabular}{|c|c|c|c|c|c|c|c|c|c|c|}
\hline & $\begin{array}{l}\text { CRITÉRIO DE } \\
\text { COMPETITIIDADE } \\
\end{array}$ & $\begin{array}{c}\text { INDICADOR DE } \\
\text { COMPETTIYIDADE } \\
\end{array}$ & DESCRIÇ̄̃o & FÓRMULA DE CáLCULO & $\begin{array}{c}\text { PERIODICIDADE } \\
\text { DE MEDICĀO }\end{array}$ & $\begin{array}{l}\text { UNIDADE DE } \\
\text { MEDIDA } \\
\end{array}$ & TIPO & ALVO & LES & LEI \\
\hline 1 & CONFABILIDADE & Frequîncia CPT & 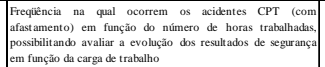 & $\frac{\sum C P T}{h H} \times 10^{6}$ & mensal & $\mathrm{tx}$ & MENOR É MELHOR & 0.75 & 0.83 & \\
\hline 2 & CONFABBLLDADE & Acidentes CPT & Indica as ocorrências de acidentes CPT (com afastamento) & $\sum C P T$ & mensal & $\mathrm{n}^{\circ}$ & MENOR É MELHOR & 0.00 & 0.00 & \\
\hline 3 & QUALIDADE & Reclamą̧ōes de Clientes & 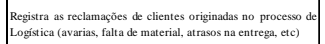 & $\sum R C$ & mensal & $\mathrm{n}^{\circ}$ & MENOR É MELHOR & 63.00 & 69.30 & \\
\hline 4 & custo & Cesta Padrăo de Fretes & 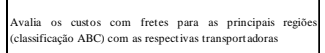 & $\frac{\sum \text { FRETE }}{\sum \text { VOLUME EMBARCADO }}$ & mensal & $\mathrm{RS} / \mathrm{t}$ & NOMINAL É MELHOR & 64.25 & 70.68 & 57.83 \\
\hline 5 & custo & Custo Operacional & 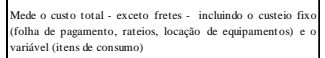 & $\frac{\sum \text { CUSTO OPERACIONAL }}{\sum \text { VOLUME EMBARCADO }}$ & mensal & $\mathrm{RS} / \mathrm{t}$ & NOMINAL É MELHOR & 16.50 & 18.15 & 14.85 \\
\hline 6 & custo & Produtividade Logisitica & 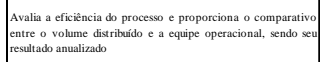 & $\frac{\sum \text { VOLUME EMBARCADO }}{\sum Q L} \times 12$ & mensal & tH/ano & NOMINAL É MELHOR & 6175.00 & 6792.50 & 5557.50 \\
\hline 7 & VELOCDDADE & $\mid \begin{array}{l}\text { Tempo Médio de } \\
\text { Permanência }\end{array}$ & 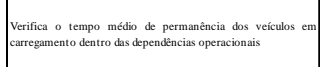 & $\frac{\sum_{i=1}^{n} t_{i} \text { VEICULOO }}{\sum \text { VEICULO }}$ & mensal & $\min$ & MENOR É MELHOR & 160.00 & 176.00 & \\
\hline 8 & VELOCDDADE & Retirada de Remessas em D+1 & \begin{tabular}{|l} 
Mensura as remessas que săo retiradus com um até un dia de \\
atraso
\end{tabular} & $\frac{\sum \text { REMESSA } D+1}{\sum \text { REMESSA }} \times 100$ & mensal & $\%$ & MAIORÉ MELHOR & 83.00 & & 74.70 \\
\hline 9 & CONFABLLIDADE & \begin{tabular}{|l} 
Entregas Atendidas Fora do \\
Prazo
\end{tabular} & $\begin{array}{l}\text { Indica as entregas realizadas fora do period programado com } \\
\text { o cliente }\end{array}$ & $\frac{\sum \text { REMESSA ATRASADA }}{\sum \text { REMESSA }}$ & mensal & $\%$ & NOMINAL É MELHOR & 4.70 & 5.17 & 4.23 \\
\hline 10 & VELOCDDADE & $\mathrm{D}+0$ & Mecde as remesesse carregatas exatamente no dia programado & $\frac{\sum R E M E S S A D+0}{\sum R E M E S S A} \times 100$ & mensal & $\%$ & MAIOR É MELHOR & 67.15 & & 60.44 \\
\hline 11 & QUALIDADE & 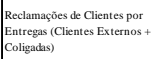 & 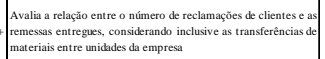 & $\frac{\sum R E C L A M A C \tilde{A} O D E C L E N T E}{\sum R E M E S S A}$ & mensal & $\mathrm{tx}$ & MENOR É MELHOR & 0.18 & 0.20 & \\
\hline 12 & CONFABBLIDADE & \begin{tabular}{|l} 
Atenđimento à Martiz de \\
Capacitaçä
\end{tabular} & \begin{tabular}{|l} 
Avaliao a atendimento às capacitaçéses previstas para a equipe \\
opperacional
\end{tabular} & $\frac{\sum \text { TREINAMENTOS REAL }}{\sum \text { TREINAMENTOS PLAN }} \times 100$ & mensal & $\%$ & NOMINAL É MELHOR & 70.00 & 77.00 & 63.00 \\
\hline 13 & FLEXIBLIDADE & \begin{tabular}{|l} 
Automanatismo de Fretes \\
vendas e Exportazia 1
\end{tabular} & 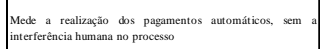 & $\frac{\sum \text { PGTO FRETE AUTO } 1}{\sum \text { PGTO FRETE }} \times 100$ & mensal & $\%$ & NOMINAL É MELHOR & 95.00 & 104.50 & 85.50 \\
\hline 14 & FLEXIBLLIDADE & $\begin{array}{l}\text { Automatismo de Fretes } \\
\text { Compras. Transferencias e } \\
\text { NF Writer } 2\end{array}$ & $\begin{array}{l}\text { Mede a reatizaça dos pagamentos automáticos, sem a } \\
\text { interferénecia humana no processo }\end{array}$ & $\frac{\sum \text { PGTO FRETE AUTO } 2}{\sum \text { PGTO FRETE }} \times 100$ & mensal & \% & NOMINAL É MELHOR & 53.00 & 58.30 & 47.70 \\
\hline 15 & VELOCDDADE & $\mathrm{D}+1$ & $\begin{array}{l}\text { Mede as remessas carregatas em até um dia após o a dataa } \\
\text { programamata }\end{array}$ & $\frac{\sum R E M E S S A D+1}{\sum R E M E S S A} \times 100$ & mensal & $\%$ & MAIOR É MELHOR & 85.00 & & 76.50 \\
\hline 16 & CONFABLLIDADE & CP Manulal de Sivata & 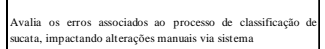 & $\frac{\sum C P M A N U A L}{\sum C P}$ & mensal & $\%$ & MENORÉ EELLHOR & 3.00 & 3.30 & \\
\hline 17 & custo & $\begin{array}{l}\text { Frete Extra de Merceado } \\
\text { Interno (Politica Comercial } \\
\text { Processo Logistico) }\end{array}$ & 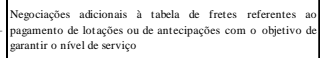 & $\frac{\sum \text { FRETE EXTRA }}{\sum \text { VOLUME EMBARCADO }}$ & mensal & $\mathrm{R} S \mathrm{t}$ & NOMINAL É MELHOR & 6.00 & 6.60 & 5.40 \\
\hline 18 & CONFABLLIDADE & $\begin{array}{l}\text { Acidentes de Cravididde A } \\
\text { SPT A A CDM A) }\end{array}$ & 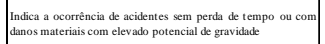 & $\sum$ ACIDENTES A & mensal & $\mathrm{n}^{\circ}$ & MENORÉ EELHOR & 1.00 & 1.00 & \\
\hline 19 & QUALIDADE & $\begin{array}{l}\text { Registro de Clientes Pendente } \\
\text { a mais de } 10 \text { dias }\end{array}$ & $\begin{array}{l}\text { Indica o numero de reclamaắeses de clientes näo resolvidas } \\
\text { dentro de } 10 \text { dias }\end{array}$ & $\sum R C^{\prime} s \geq 10$ DIAS & mensal & $\mathrm{n}^{\circ}$ & MENORÉ MELLHOR & 0.00 & 1.00 & \\
\hline 20 & FLEXIBLIDADE & Mult-modalidiade & Avalia a composiçăo modal das entregas & $\frac{\sum R E M E S S A S M U L T T}{\sum R E M E S S A S}$ & mensal & $\%$ & MAIOR É MELHOR & 25.00 & & 22.50 \\
\hline 21 & FLEXIBLLIDADE & оTIF & $\begin{array}{l}\text { Mede a performance de atendimento total no prazo dos } \\
\text { pecuidos }\end{array}$ & $\frac{\sum \text { REMESSAS OTIF }}{\sum R E M E S S A S}$ & mensal & $\%$ & NOMINAL É MELHOR & 87.00 & 95.70 & 78.30 \\
\hline 22 & custo & $\begin{array}{l}\text { Relaçăo entre Frete e Receita } \\
\text { Liquida }\end{array}$ & 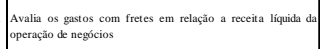 & $\frac{\sum \text { FRETE REAL }}{\sum \text { RECEITA LIOUUIDA }}$ & mensal & $\mathrm{tx}$ & NOMINAL É MELHOR & 3.00 & 3.30 & 2.70 \\
\hline 23 & VELOCDDADE & Nota Fiscal Automática & Mede a performance da automatizą̧io dos processos & $\frac{\sum N F A U T O}{\sum N F} \times 100$ & mensal & $\%$ & NOMINAL É MELHOR & 61.00 & 67.10 & 54.90 \\
\hline 24 & custo & Cesta Básica de Fretes & Mede os fretes cotados e contratados para as principais reğiōes & $\frac{\sum \text { FRETE CESTA }}{\sum \text { VILUME EMBARCADO }}$ & mensal & $\mathrm{R} S / \mathrm{t}$ & MENORÉ EELLHOR & 52.00 & 57.20 & \\
\hline 25 & custo & $\begin{array}{l}\text { Variaçăo da Cesta Padrão de } \\
\text { Fretes }\end{array}$ & Avalia a variagăo d cesta Básica de fretes & $\frac{\sum \text { FRETE REAL }}{\sum \text { FRETE PLAN }} \times 100$ & mensal & $\%$ & MENOR É MELHOR & 0.00 & 1.10 & \\
\hline 26 & CONFABLLIDADE & $\begin{array}{l}\text { Total de Remessas Faturachs } \\
\text { em D } 23\end{array}$ & $\mid \begin{array}{l}\text { Mede as remessas carregahas } \mathrm{em} 3 \text { ou mais dias alkm do } \\
\text { planejado }\end{array}$ & $\frac{\sum R E M E S S A D \geq 3}{\sum R E M E S S A} \times 100$ & mensal & $\%$ & MENOR É MELHOR & 2.50 & 2.75 & \\
\hline 27 & custo & Frete Real & Mede a integraçăo de todas as componentes de frete & $\frac{\sum \text { FRETE REAL }}{\sum \text { VOLUME EMBARCADO }}$ & mensal & $\mathrm{RS} / \mathrm{t}$ & NOMINAL É MELHOR & 81.81 & 89.99 & 73.63 \\
\hline 28 & custo & Orçamento Matricial & \begin{tabular}{|l} 
Mede o gasto realizado em relą̧äo aos centros de custo \\
pertencentes à areas
\end{tabular} & $\sum$ GASTOS & mensal & RS & NOMINAL É MELHOR & 4850.00 & 5335.00 & 4365.00 \\
\hline
\end{tabular}

Revista Produção Online, Florianópolis, SC, v.12, n. 3, p. 756-778, jul./set. 2012. 
ANEXO 2 - IGP para os meses de janeiro/09 à junho/09

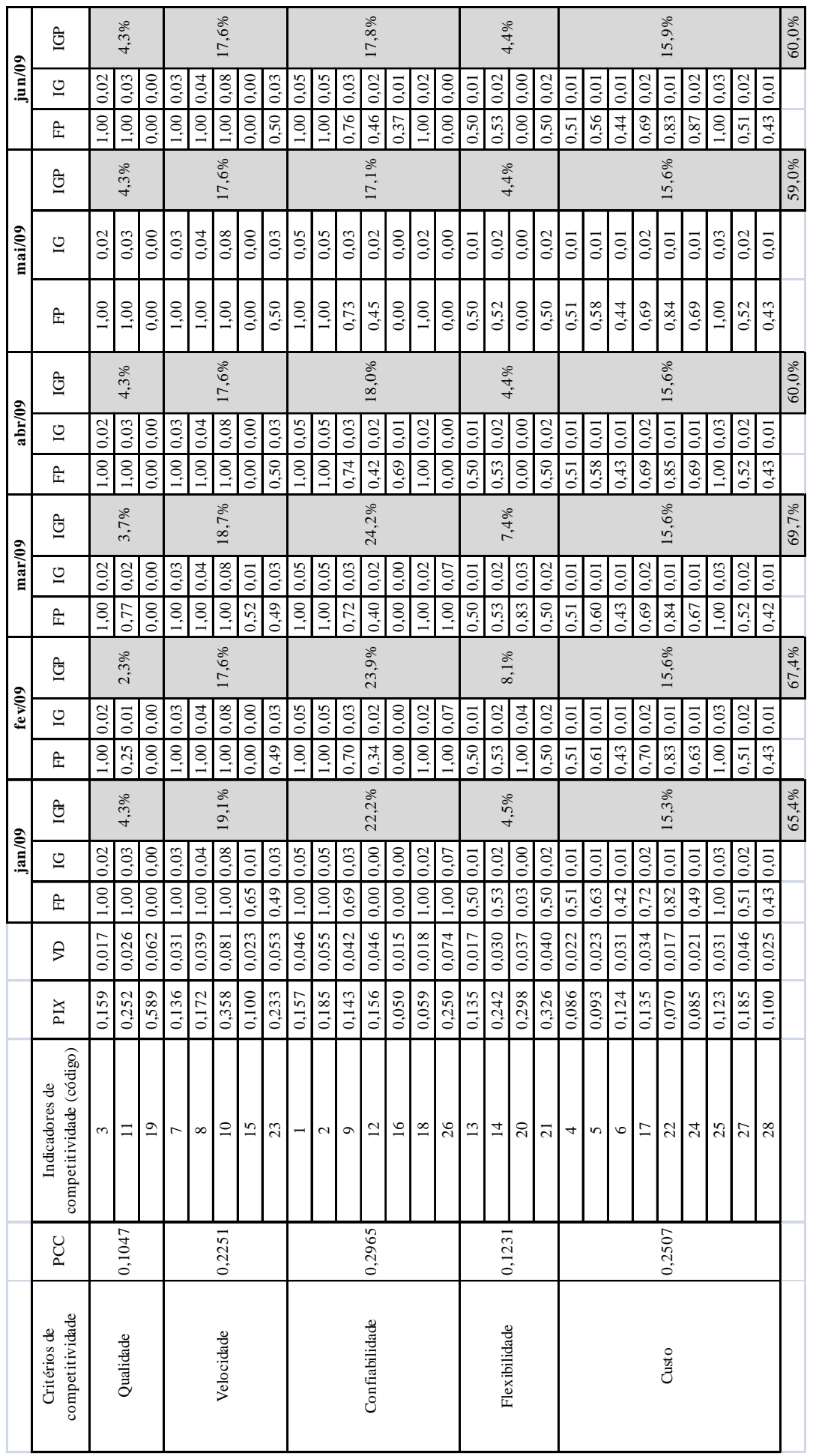

Revista Produção Online, Florianópolis, SC, v.12, n. 3, p. 756-778, jul./set. 2012. 